\title{
Superheating fields of superconductors: Asymptotic analysis and numerical results
}

\author{
Andrew J. Dolgert1, S. John Di Bartold2, and Alan T. Dorsey 3 \\ Department of Physics, University of Virginia, McCormick Road, Charlottesville, Virginia 22901
}

(February 23, 2018)

\begin{abstract}
The superheated Meissner state in type-I superconductors is studied both analytically and numerically within the framework of Ginzburg-Landau theory. Using the method of matched asymptotic expansions we have developed a systematic expansion for the solutions of the Ginzburg-Landau equations in the limit of small $\kappa$, and have determined the maximum superheating field $H_{\text {sh }}$ for the existence of the metastable, superheated Meissner state as an expansion in powers of $\kappa^{1 / 2}$. Our numerical solutions of these equations agree quite well with the asymptotic solutions for $\kappa<0.5$. The same asymptotic methods are also used to study the stability of the solutions, as well as a modified version of the Ginzburg-Landau equations which incorporates nonlocal electrodynamics. Finally, we compare our numerical results for the superheating field for large- $\kappa$ against recent asymptotic results for large- $\kappa$, and again find a close agreement. Our results demonstrate the efficacy of the method of matched asymptotic expansions for dealing with problems in inhomogeneous superconductivity involving boundary layers.
\end{abstract}

PACS numbers: 74.40.+k, 74.55.+h, 74.60.Ec

\section{INTRODUCTION}

In equilibrium, the superconducting Meissner phase of a bulk type-I superconducting sample exists below a thermodynamic critical field $H_{c}$ (or below $H_{c 1}$ in a type-II superconductor); above this field the sample reverts to the normal phase (or the flux lattice phase in a type-II superconductor). However, because the phase transition in both cases is first-order it is possible to superheat the Meissner phase and delay the transition to fields well above $H_{c}$ or $H_{c 1}$. This superheated, metastable Meissner phase is eventually destroyed at a maximum superheating field $H_{\text {sh }}$. Understanding the origin and stability of the superheated state is the first step in providing a complete description of the time-dependent collapse of the Meissner phase, which is important for many applications of type-I superconductors. For instance, there have been recent proposals to use superheated type-I superconductors as detectors for elementary particles, so that the sample acts as a superconducting "bubble chamber" [1]. The passage of a sufficiently energetic particle through the sample would initiate the transition to the normal state. Measuring the superheating field also provides one of the few methods of experimentally determining the Ginzburg-Landau parameter $\kappa$ in type-I superconductors [2].

The precise value of the maximum superheating field may depend upon extrinsic factors such as defects in the sample and sample preparation and geometry. If these effects can be minimized then the limit of superheating is determined by the boundaries and geometry of the sample. The simplest and most widely studied geometry is a superconducting half-space with a magnetic field applied parallel to the surface of the superconductor, which is the geometry considered in the remainder of this paper. To model the superconductor we will use the Ginzburg-Landau (GL) equations, which provide an accurate description of the surface behavior provided the coherence length $\xi$ is large compared to microscopic length scales [3]. Previous studies of superheating in type-I superconductors have used a variety of heuristic methods to determine the behavior of the GL equations near the surface. Ginzburg [4] inferred the leading $\kappa^{-1 / 2}$ dependence of the superheating field from the form of the Ginzburg-Landau equations. Apparently unaware of Ginzburg's work, the Orsay group [5] used a variational argument to show that $H_{\mathrm{sh}} / H_{c} \approx 2^{-1 / 4} \kappa^{-1 / 2}$. By combining an ingenious guess for the behavior of the superconducting order parameter near the surface with a variational calculation, Parr [6] was able to calculate the next order correction to the Orsay group's result. In addition to this analytical work there has also been a great deal of numerical work on solving the GL equations in small- $\kappa$ limit [7 10], which is reviewed in Ref. [11]. The numerical results appear to confirm at least some of the analytical work, although admittedly over a somewhat restricted range of $\kappa$. One deficiency common to all of the previous analytical approaches is an $a d-h o c$ construction of approximate solutions of the GL equations, leaving us without a procedure for systematically improving upon these approximations. In addition, the issue of the stability of the solutions in the small- $\kappa$ limit seems not to have been addressed rigorously (for one attempt see [12]).

In this paper we re-examine the problem of superheating in type-I superconductors by using the method of matched asymptotic expansions [13,14] to solve the GL equations in the small- $\kappa$ limit. This method was originally developed to treat boundary layer problems in fluid mechanics [14] in a controlled and systematic fashion, and is particularly 
well suited to the superheating problem, as all of the technical difficulties arise due to a "boundary layer" at the surface. Using this method we can calculate the superheating field in the small- $\kappa$ limit as an asymptotic expansion in powers of $\kappa^{1 / 2}$, construct uniform asymptotic expansions (i.e., expansions valid for all $x$ as $\kappa \rightarrow 0$ ) for the order parameter and magnetic field, determine the stability of the solutions, and treat nonlocal electrodynamic effects. Where appropriate we compare our asymptotic results against numerical solutions of the GL equations, and we generally find excellent agreement. We have chosen to present our results in detail, as the methods are probably unfamiliar to most physicists. Matched asymptotic expansions have recently been used by Chapman [15] to study superheating in the large- $\kappa$ limit, and our results complement his work. This paper, taken together with Chapman's work, demonstrates that these perturbation methods can provide a powerful calculational tool for solving problems in inhomogeneous or nonequilibrium superconductivity.

The remainder of this paper is organized as follows. In Sec. II we describe our numerical methods for solving the GL equations. In Sec. III we develop the method of matched asymptotic expansions for the solution of the GL equations in the small- $\kappa$ limit, and determine the first three terms in the expansion of $H_{\mathrm{sh}}$ in powers of $\kappa^{1 / 2}$. In addition, we construct uniform expansions for the order parameter and magnetic field and compare them against our asymptotic results. In Sec. IV we examine the second variation of the GL free energy, $\delta^{2} \mathcal{F}$, in order to determine the stability of our solutions. This is done for both one and two-dimensional perturbations. In Sec. V the method is generalized to treat nonlocal electrodynamics. Section VII compares Chapman's asymptotic expansion for $H_{\text {sh }}$ for large- $\kappa$ with our numerical results, and we find remarkably good agreement. Finally, Sec. VIII is a summary and discussion of our results.

\section{NUMERICAL METHODS}

The GL free energy of a superconducting sample occupying the half-space $x>0$ is

$$
\mathcal{F}[f, q]=\int_{x>0} d^{3} r\left[\frac{1}{\kappa^{2}}(\nabla f)^{2}+\frac{1}{2}\left(1-f^{2}\right)^{2}+f^{2} \mathbf{q}^{2}+\left(\mathbf{H}_{a}-\nabla \times \mathbf{q}\right)^{2}\right]
$$

where $\kappa$ is the GL parameter, $f$ is the amplitude of the superconducting order parameter, $\mathbf{q}$ is the gauge-invariant vector potential $(\mathbf{h}=\nabla \times \mathbf{q})$, and $\mathbf{H}_{a}$ is the applied magnetic field. The lengths are in units of the penetration depth $\lambda$ and fields are in units of $\sqrt{2} H_{c}$. Minimizing this expression with respect to both $f$ and $\mathbf{q}$ results in the GL equations. In one dimension, with $f=f(x)$ and $\mathbf{q}=(0, q(x), 0)$, these equations are

$$
\begin{gathered}
\frac{1}{\kappa^{2}} f^{\prime \prime}-q^{2} f+f-f^{3}=0, \\
q^{\prime \prime}-f^{2} q=0, \\
h=q^{\prime} .
\end{gathered}
$$

The task at hand is to solve these equations numerically for a superconducting half-space and to find the largest possible applied field $\left(H_{\mathrm{sh}}\right)$ which permits a superconducting solution. To insure that no current passes through the boundary at $x=0$ and that the sample is totally superconducting infinitely far from the surface, we impose the boundary conditions

$$
f^{\prime}(0)=0, \quad f(x) \rightarrow 1 \text { as } x \rightarrow \infty .
$$

Since the field at the surface must equal the applied field $H_{a}$, and the field infinitely far from the surface must equal 0 , we impose the boundary conditions

$$
h(0)=H_{a}, \quad q(x) \rightarrow 0 \text { as } x \rightarrow \infty .
$$

For $\kappa \rightarrow 0$, we rescale the equations as $x^{\prime}=\kappa x$ making the new unit of length the correlation length $\xi$. Since $\xi \gg \lambda$ in this limit, a numerical solution over a domain much larger than $\xi$ would insure that the regions of rapid change for $f$ and $h$ would be included. (For small $\kappa$, we find that solving for $x^{\prime}<500$ is sufficient.) In the large $\kappa$ limit, we use the rescaled equations again, but we increase the size of the domain depending on the value of $\kappa$. (The equations must be solved for domains as large as $x^{\prime}<10^{4}$ for values of $\kappa \sim 10^{3}$.) 
The equations can be solved using the relaxation method $\sqrt{16}$. By replacing these ordinary differential equations with finite difference equations, one can start with a guess to the solution and iterate using a multi-dimensional Newton's method until it relaxes to the true solution. In order to more accurately pick up the detail near the boundary, we choose a grid of discrete points with a higher density near $x=0$. In particular we choose a density which roughly varies as the inverse of the distance from the boundary. (For low $\kappa$ our density, in units of mesh points per coherence length, varies approximately from $10^{7}$ near the boundary to $10^{3}$ at the farthest point from the boundary, while for high $\kappa$ it varies from $10^{5}$ to $10^{-2}$.)

$H_{\text {sh }}$ can be found in the following way. For a given value of $\kappa$ an initial guess is made where there is no applied field and the sample is completely superconducting ( $f \equiv 1, q \equiv 0, h \equiv 0)$. The field $H_{a}$ is then "turned up" in small increments. For each value of $H_{a}$ a solution is sought using the result from the previous lower field solution as an initial guess. Eventually a maximum value for $H_{a}$ is reached, above which one of two things happens: our algorithm fails to converge to a solution or it converges to the normal (nonsuperconducting solution). This maximum value of $H_{a}$ is the numerical result for $H_{\mathrm{sh}}$. Using this algorithm, $H_{\mathrm{sh}}(\kappa)$ can be found for a wide range of $\kappa$ 's. Each run (for a given $\kappa$ ) takes about $60 \mathrm{cpu}$ minutes on an IBM RS 6000/370. We find it sufficient for the purposes of this paper to deal with superheating field values for $10^{-3}<\kappa<10^{3}$.

\section{ASYMPTOTIC EXPANSIONS FOR SMALL- $\kappa$}

In this section we will develop an asymptotic expansion for the superheating field for small- $\kappa$, using the method of matched asymptotic expansions 13,14. For small- $\kappa$ the dominant length scale is the coherence length $\xi$, so it is natural to have $\xi$ serve as our unit of length. This is achieved by rescaling $x$ by $\kappa$, introducing a new dimensionless coordinate $x^{\prime}=\kappa x$. The resulting GL equations in these "outer variables" are

$$
\begin{gathered}
f^{\prime \prime}-q^{2} f+f-f^{3}=0, \\
\kappa^{2} q^{\prime \prime}-f^{2} q=0, \\
h=\kappa q^{\prime},
\end{gathered}
$$

with the primes now denoting differentiations with respect to $x^{\prime}$.

Outer solution. In order to obtain the outer solutions expand $f, q$, and $h$ in powers of $\kappa$ :

$$
\begin{aligned}
& f=f_{0}+\kappa f_{1}+\kappa^{2} f_{2}+\ldots, \\
& q=q_{0}+\kappa q_{1}+\kappa^{2} q_{2}+\ldots, \\
& h=h_{0}+\kappa h_{1}+\kappa^{2} h_{2}+\ldots .
\end{aligned}
$$

Substituting into Eqs. (3.1)-(3.3), at $O(1)$ we have

$$
\begin{gathered}
f_{0}^{\prime \prime}-q_{0}^{2} f_{0}+f_{0}-f_{0}^{3}=0, \\
-f_{0}^{2} q_{0}=0 .
\end{gathered}
$$

Since we want $f \rightarrow 1$ as $x^{\prime} \rightarrow \infty$, the only possible solution to Eq. (3.8) is $q_{0}=0$. We can then immediately integrate Eq. (3.7),

$$
f_{0}\left(x^{\prime}\right)=\tanh \left(\frac{x^{\prime}+x_{0}}{\sqrt{2}}\right)
$$

with $x_{0}$ an arbitrary constant. To $O(\kappa)$, the outer equations are

$$
\begin{gathered}
f_{1}^{\prime \prime}-2 q_{0} f_{0} q_{1}-q_{0}^{2} f_{1}+f_{1}-3 f_{0}^{2} f_{1}=0, \\
-f_{0}^{2} q_{1}-2 f_{0} q_{0} f_{1}=0
\end{gathered}
$$




$$
h_{0}=0 .
$$

Once again, the only solution to Eq. (3.11) is $q_{1}=0$; substituting this into Eq. (3.10), we find $f_{1}=C_{1} f_{0}^{\prime}$, with $C_{1}$ a constant:

$$
f_{1}=\frac{C_{1}}{\sqrt{2}} \operatorname{sech}^{2}\left(\frac{x^{\prime}+x_{0}}{\sqrt{2}}\right)
$$

We can continue in this manner; at every order $q_{n}=0, h_{n}=0$, and $f_{n}=C_{n} f_{0}^{(n)}$, with the $C_{n}$ 's constants which are determined by matching onto the inner solution.

Inner solution. The outer solution breaks down within a boundary layer of $O(\kappa)$ near the surface. This suggests introducing a rescaled inner coordinate $X=x^{\prime} / \kappa$, so that $X=O(1)$ within the boundary layer. It is also possible to rescale $f$ and $q$, with the hope that this will lead to a tractable inner problem. Such a rescaling must lead to a successful matching of the inner and outer solutions; i.e., the inner solutions as $X \rightarrow \infty$ must match onto the outer solutions as $x^{\prime} \rightarrow 0$. Since $f_{0}(0)=\tanh \left(x_{0} / \sqrt{2}\right)$, then assuming that $x_{0} \neq 0$ we have $f_{0}(0)=O(1)$, indicating that the order parameter should not be rescaled in the inner region; therefore we set $f\left(x^{\prime}\right)=F(X)$ in the inner region. However, from the outer solution for the vector potential we see that the only constraint on $q(X)$ in the inner region is that $q(X) \rightarrow 0$ as $X \rightarrow \infty$ (presumably exponentially). Therefore, we are free to rescale $q$ by $\kappa$ in the inner region, hopefully in a way which simplifies the inner equations. One possibility is $q\left(x^{\prime}\right)=\kappa^{-\alpha} Q(X)$; substituting this into the GL equations, Eqs. (3.1)-(3.3), we see that unless $2 \alpha$ is an integer, fractional powers of $\kappa$ will be introduced into the inner equations, contradicting our expansion of $f$ and $q$ in integer powers of $\kappa$ in the outer region. Therefore, the simplest assumption is that $\alpha=1 / 2$, leading to the following choice for the inner variables:

$$
x^{\prime}=\kappa X, \quad f\left(x^{\prime}\right)=F(X), \quad q\left(x^{\prime}\right)=\kappa^{-1 / 2} Q(X), \quad h\left(x^{\prime}\right)=H(X) .
$$

In these variables Eqs. (3.1)-(3.3) become

$$
\begin{gathered}
F^{\prime \prime}-\kappa Q^{2} F+\kappa^{2}\left(F-F^{3}\right)=0, \\
Q^{\prime \prime}-F^{2} Q=0, \\
\kappa^{1 / 2} H=Q^{\prime},
\end{gathered}
$$

where now the primes denote differentiation with respect to $X$. The boundary conditions are

$$
F^{\prime}(0)=0, \quad H(0)=H_{a} .
$$

The next step is to expand the inner solutions in powers of $\kappa$ :

$$
\begin{gathered}
F=F_{0}+\kappa F_{1}+\kappa^{2} F_{2}+\ldots, \\
Q=Q_{0}+\kappa Q_{1}+\kappa^{2} Q_{2}+\ldots, \\
H=\kappa^{-1 / 2} H_{0}+\kappa^{1 / 2} H_{1}+\ldots
\end{gathered}
$$

Note that there is no term of $O(1)$ in the expansion for $H$, since we would be unable to match such a term to the outer solution. Using the boundary condition $H(0)=H_{a}$ leads to

$$
H_{a}=\kappa^{-1 / 2} H_{0}(0)+\kappa^{1 / 2} H_{1}(0)+\ldots
$$

Substituting these expansions into Eqs. (3.15)-(3.17), at $O(1)$ we obtain

$$
F_{0}^{\prime \prime}=0, \quad Q_{0}^{\prime \prime}-F_{0}^{2} Q_{0}=0, \quad H_{0}=Q_{0}^{\prime} .
$$

Solving these equations subject to the boundary conditions (3.18) (we also need $Q_{0} \rightarrow 0$ as $x \rightarrow \infty$ in order to match onto the outer solution), we obtain

$$
F_{0}(X)=A_{0}, \quad Q_{0}(X)=B_{0} e^{-A_{0} X}, \quad H_{0}(0)=-A_{0} B_{0},
$$


with $A_{0}$ and $B_{0}$ constants. In what follows we will assign $F_{n}(0)=A_{n}$ and $Q_{n}(0)=B_{n}$ for notational simplicity. The $O(\kappa)$ equations are

$$
F_{1}^{\prime \prime}=Q_{0}^{2} F_{0}, \quad Q_{1}^{\prime \prime}-F_{0}^{2} Q_{1}=2 F_{0} Q_{0} F_{1}, \quad H_{1}=Q_{1}^{\prime} .
$$

Solving with the boundary condition $F_{1}^{\prime}(0)=0$, we obtain

$$
\begin{aligned}
& F_{1}(X)=A_{1}+\frac{B_{0}^{2}}{4 A_{0}}\left[2 A_{0} X+e^{-2 A_{0} X}-1\right], \\
& Q_{1}(X)=e^{-A_{0} X}\left\{B_{1}-\frac{B_{0}^{3}}{16 A_{0}^{2}}\left[1-e^{-2 A_{0} X}\right.\right. \\
& \left.\left.+16 \frac{A_{0}^{2} A_{1}}{B_{0}^{2}} X+4 A_{0}^{2} X^{2}\right]\right\}, \\
& H_{1}(0)=-\frac{1}{8} \frac{B_{0}^{3}}{A_{0}}-A_{0} B_{1}-A_{1} B_{0} .
\end{aligned}
$$

Finally, to $O\left(\kappa^{2}\right)$ we have for $F_{2}$

$$
F_{2}^{\prime \prime}=-F_{0}+F_{0}^{3}+2 Q_{0} Q_{1} F_{0}+Q_{0}^{2} F_{1},
$$

the solution of which (with $\left.F_{2}^{\prime}(0)=0\right)$ is

$$
\begin{aligned}
F_{2}(X)=\frac{17}{128} & \frac{B_{0}^{4}}{A_{0}^{3}}+\frac{1}{4} \frac{B_{0}^{2} A_{1}}{A_{0}^{2}}-\frac{1}{2} \frac{B_{0} B_{1}}{A_{0}}+A_{2}+\left(B_{0} B_{1}-\frac{3}{32} \frac{B_{0}^{4}}{A_{0}^{2}}\right) X-\frac{1}{2} A_{0}\left(1-A_{0}^{2}\right) X^{2} \\
+ & {\left[\frac{1}{2} \frac{B_{0} B_{1}}{A_{0}}-\frac{1}{4} \frac{B_{0}^{2} A_{1}}{A_{0}^{2}}-\frac{5}{32} \frac{B_{0}^{4}}{A_{0}^{3}}-\left(\frac{1}{8} \frac{B_{0}^{4}}{A_{0}^{2}}+\frac{1}{2} \frac{B_{0}^{2} A_{1}}{A_{0}}\right) X-\frac{1}{8} \frac{B_{0}^{4}}{A_{0}} X^{2}\right] e^{-2 A_{0} X} } \\
& +\frac{3}{128} \frac{B_{0}^{4}}{A_{0}^{3}} e^{-4 A_{0} X} .
\end{aligned}
$$

The expression for $Q_{2}$ is even more unwieldy, and is not needed in what follows.

Matching. To determine the various integration constants which have been introduced we must match the inner solution to the outer solution. Since the outer solution for $q$ is simply $q=0$, and all of our inner solutions decay exponentially for large $X$, the matching is automatically satisfied for $q$, as well as for the magnetic field $h$. To match the inner and outer solutions for the order parameter, we are guided by the van Dyke matching principle [14], which states that the $m$ term inner expansion of the $n$ term outer solution should match onto the $n$ term outer expansion of the $m$ term inner solution. In our case we will take $m=3$ and $n=2$. Therefore, write the two term outer solution $f_{0}\left(x^{\prime}\right)+\kappa f_{1}\left(x^{\prime}\right)$ in terms of the inner variable $X$, and expand for small $\kappa$, keeping the first three terms in the expansion in powers of $\kappa$ :

$$
\begin{aligned}
f_{0}(\kappa X)+\kappa f_{1}(\kappa X) \sim \tanh & \left(\frac{x_{0}}{\sqrt{2}}\right)+\kappa \operatorname{sech}^{2}\left(\frac{x_{0}}{\sqrt{2}}\right) \frac{1}{\sqrt{2}}\left[C_{1}+X\right] \\
& +\kappa^{2} \operatorname{sech}^{2}\left(\frac{x_{0}}{\sqrt{2}}\right) \tanh \left(\frac{x_{0}}{\sqrt{2}}\right)\left[-C_{1} X-\frac{X^{2}}{2}\right] .
\end{aligned}
$$

Next, write the three term inner solution $F_{0}(X)+\kappa F_{1}(X)+\kappa^{2} F_{2}(X)$ in terms of the outer variable $x^{\prime}$, and expand for small $\kappa$, this time keeping the first two terms of the expansion:

$$
\begin{aligned}
F_{0}\left(x^{\prime} / \kappa\right)+\kappa F_{1}\left(x^{\prime} / \kappa\right)+\kappa^{2} F_{2}\left(x^{\prime} / \kappa\right) \sim A_{0}+ & \frac{B_{0}^{2}}{2} x^{\prime}-\frac{1}{2} A_{0}\left(1-A_{0}^{2}\right) x^{\prime 2} \\
& +\kappa\left[A_{1}-\frac{B_{0}^{2}}{4 A_{0}}+\left(B_{0} B_{1}-\frac{3}{32} \frac{B_{0}^{4}}{A_{0}^{2}}\right) x^{\prime}\right] .
\end{aligned}
$$

By writing both expressions in terms of $x^{\prime}$, and equating the various coefficients of $x^{\prime}$ and $\kappa$, we see that the expansions do indeed match if we choose 


$$
\begin{gathered}
A_{0}=\tanh \left(\frac{x_{0}}{\sqrt{2}}\right), \\
B_{0}=-2^{1 / 4} \operatorname{sech}\left(\frac{x_{0}}{\sqrt{2}}\right)=-2^{1 / 4}\left(1-A_{0}^{2}\right)^{1 / 2} \\
A_{1}=\frac{B_{0}^{2}}{4 A_{0}}+\operatorname{sech}^{2}\left(\frac{x_{0}}{\sqrt{2}}\right) \frac{C_{1}}{\sqrt{2}}=\frac{\sqrt{2}}{4} \frac{1-A_{0}^{2}}{A_{0}}+\left(1-A_{0}^{2}\right) \frac{C_{1}}{\sqrt{2}}, \\
B_{1}=\frac{3}{32} \frac{B_{0}^{3}}{A_{0}^{2}}-\frac{\sqrt{2} A_{0}\left(1-A_{0}^{2}\right)}{B_{0}} \frac{C_{1}}{\sqrt{2}} .
\end{gathered}
$$

Eliminating $C_{1}$,

$$
B_{1}=-\frac{\sqrt{2} A_{0} A_{1}}{B_{0}}+\frac{3}{32} \frac{B_{0}^{3}}{A_{0}^{2}}+\frac{1}{2} \frac{1-A_{0}^{2}}{B_{0}} .
$$

Substituting into our expressions for $H_{0}(0)$ and $H_{1}(0)$ from Eqs. (3.24) and (3.28), we obtain

$$
\begin{gathered}
H_{0}(0)=2^{1 / 4} A_{0}\left(1-A_{0}^{2}\right)^{1 / 2}, \\
H_{1}(0)=\frac{2^{3 / 4}}{64} \frac{\left(2 A_{0}^{2}+14\right)\left(1-A_{0}^{2}\right)^{1 / 2}}{A_{0}}-\frac{2^{1 / 4}\left(2 A_{0}^{2}-1\right)}{\left(1-A_{0}^{2}\right)^{1 / 2}} A_{1} .
\end{gathered}
$$

In order to calculate the superheating field (or, more correctly, the maximum superheating field), we need to maximize $H_{0}(0)$ and $H_{1}(0)$ with respect to $A_{0}$ and $A_{1}$. Maximizing $H_{0}(0)$ with respect to $A_{0}$, we find that the maximum occurs at $A_{0}^{*}=1 / \sqrt{2}, B_{0}^{*}=-2^{-1 / 4}$, so that $H_{0}(0)=2^{-3 / 4}$. Substituting this result into $H_{1}(0)$, we find the surprising result that the coefficient of $A_{1}$ is zero, and $H_{1}(0)=2^{3 / 4} 15 / 64$. Our superheating field is then

$$
H_{\mathrm{sh}}=2^{-3 / 4} \kappa^{-1 / 2}\left[1+\frac{15 \sqrt{2}}{32} \kappa+O\left(\kappa^{2}\right)\right] .
$$

In order to determine $A_{1}$ we need to proceed to a higher order calculation. The method is the same as before, although the algebra quickly becomes tedious; we have used the computer algebra system Maple $V$ to organize the expansion. The results from a six term inner expansion are summarized in Table $\mathbb{\|}$. Including the next order term in the expansion in the superheating field, we have

$$
H_{\mathrm{sh}}=2^{-3 / 4} \kappa^{-1 / 2}\left[1+\frac{15 \sqrt{2}}{32} \kappa-\frac{325}{1024} \kappa^{2}+O\left(\kappa^{3}\right)\right] .
$$

The first term is exactly the result obtained by the Orsay group [4.5], who used a variational argument to obtain their result. The second term is identical to the result obtained by Parr [6]. Parr combined an inspired guess for the behavior of the order parameter near the surface with a variational calculation in order to obtain his result. It is interesting to note that our result for $A_{1}$ also agrees with Parr's result. The advantage of the method of matched asymptotic expansions is that we can make this expansion systematic, and therefore in principle carry out this expansion as far as we wish. The third term in Eq. (3.41) is one of the new results of this paper; the fourth and fifth terms are included in Table [. With the five-term expansion for $H_{\mathrm{sh}}$ it is possible to employ resummation techniques to improve the expansion. For instance, the [2,2] Padé approximant [13] is

$$
H_{\mathrm{sh}}^{\text {Pade }}=2^{-3 / 4} \kappa^{-1 / 2} \frac{1+5.4447812 \kappa+4.2181012 \kappa^{2}}{1+4.7818686 \kappa+1.3655230 \kappa^{2}} .
$$

In Fig. 1 we compare the numerically calculated superheating field against the one, two, and three term asymptotic expansions. The one term (i.e., the Orsay group) result never seems particularly accurate. There is a marked improvement with the two term expansion, with the three term expansion offering only a modest additional improvement. The $[2,2]$ Padé approximant agrees with the numerical data to within about $1 \%$ all the way to $\kappa=1$. 
Uniform solutions. From the inner and outer expansions it is possible to construct uniform solutions, which are asymptotically correct for all $x$ as $\kappa \rightarrow 0$. To do this we simply add the inner and outer solutions of a given order, which guarantees the correct behavior in the outer region as well as in the boundary layer. However, this would produce a result which was $2 f_{\text {match }}$ in the matching region, so we need to subtract $f_{\text {match }}$ in order to obtain the correct behavior in this region. As an example, we will construct the 2-term uniform solution for the order parameter. Adding the two-term outer solution, $f_{0}\left(x^{\prime}\right)+\kappa f_{1}\left(x^{\prime}\right)$, to the two-term inner solution, $F_{0}(X)+\kappa F_{1}(X)$, subtracting the solution in the matching region, which is $1 / \sqrt{2}+(\sqrt{2} / 4) \kappa X-(15 / 32) \kappa$, and writing the entire combination in terms of the original variable $x$ (which is the same as $X$ ), we obtain

$$
f_{\text {unif }, 2}(x)=\tanh \left(\frac{\kappa x+x_{0}}{\sqrt{2}}\right)-\frac{15}{16} \kappa \operatorname{sech}^{2}\left(\frac{\kappa x+x_{0}}{\sqrt{2}}\right)+\frac{\kappa}{4} e^{-\sqrt{2} x} .
$$

As $x \rightarrow \infty, f_{\text {unif }, 2}(x) \rightarrow 1$; also, $f_{\text {unif }, 2}(0)=1 / \sqrt{2}-(7 / 32) \kappa$, as we expect. However, $f_{\text {unif }, 2}^{\prime}(0)=(15 / 64) \kappa^{2}$, so that the zero-derivative boundary condition is only satisfied to $O(\kappa)$.

In Fig. 2 and Fig. 3 we compare the numerically calculated order parameter and magnetic field with the two term outer solutions and the three term inner solutions. The agreement is quite good for $\kappa=0.1$, with deviations appearing at $\kappa=0.5$. These figures also illustrate the existence of a matching region where the inner and outer solutions overlap; this region grows as $\kappa \rightarrow 0$. Lastly, we show in Fig. A how the two term uniform expansion constructed earlier supplies a uniform approximation to the order parameter and magnetic field over the whole region.

\section{STABILITY ANALYSIS OF THE SOLUTIONS}

Having obtained an asymptotic expansion for the superheating field $H_{\mathrm{sh}}$ in powers of $\kappa^{1 / 2}$, we now examine the stability of the solution with respect to infinitesimal perturbations by studying the second variation of the free energy, $\delta^{2} \mathcal{F}$. Perturbations with $\delta^{2} \mathcal{F}>0$ correspond to stable solutions, while those with $\delta^{2} \mathcal{F}<0$ correspond to unstable solutions. We will again use the method of matched asymptotics to solve for the eigenfunctions of the linear stability operator. We first determine the stability in the simpler one-dimensional situation then we discuss the two-dimensional case.

\section{A. Stability with respect to one-dimensional perturbations}

If we perturb the extremal solution $(f, q)$ of the GL equations by allowing $f(x) \rightarrow f(x)+\tilde{f}(x)$ and $q(x) \rightarrow q(x)+\tilde{q}(x)$, then the second variation of the free energy functional is

$$
\delta^{2} \mathcal{F}=\int_{0}^{\infty} d x\left[\frac{1}{\kappa^{2}} \tilde{f}^{\prime 2}+\left(3 f^{2}+q^{2}-1\right) \tilde{f}^{2}+4 f q \tilde{f} \tilde{q}+f^{2} \tilde{q}^{2}+\tilde{q}^{2}\right] .
$$

The boundary conditions on $\tilde{f}$ and $\tilde{q}$ should be chosen so as to not perturb $f$ and $h$ at the surface, so that

$$
\tilde{f}^{\prime}(0)=\tilde{q}^{\prime}(0)=0, \quad \tilde{f}(\infty)=\tilde{q}(\infty)=0 .
$$

We can then integrate Eq. (4.1) by parts to obtain

$$
\delta^{2} \mathcal{F}=\int_{0}^{\infty} d x\left[\tilde{f}\left(-\frac{1}{\kappa^{2}} \frac{d^{2}}{d x^{2}}+q^{2}+3 f^{2}-1\right) \tilde{f}+\tilde{q}\left(-\frac{d^{2}}{d x^{2}}+f^{2}\right) \tilde{q}+4 q f \tilde{q} \tilde{f}\right] .
$$

This quadratic form can be conveniently written as

$$
\delta^{2} \mathcal{F}=\int_{0}^{\infty} d x(\tilde{f}, \tilde{q}) \hat{L}_{1}\left(\begin{array}{c}
\tilde{f} \\
\tilde{q}
\end{array}\right)
$$

where $\hat{L}_{1}$ is the self-adjoint linear operator

$$
\hat{L}_{1}\left(\begin{array}{c}
\tilde{f} \\
\tilde{q}
\end{array}\right)=\left(\begin{array}{cc}
-\frac{1}{\kappa^{2}} \frac{d^{2}}{d x^{2}}+q^{2}+3 f^{2}-1 & 2 f q \\
2 f q & -\frac{d^{2}}{d x^{2}}+f^{2}
\end{array}\right)\left(\begin{array}{c}
\tilde{f} \\
\tilde{q}
\end{array}\right) .
$$


In order to analyze the stability, expand $\tilde{f}$ and $\tilde{q}$ as

$$
\left(\begin{array}{c}
\tilde{f} \\
\tilde{q}
\end{array}\right)=\sum_{n} c_{n}\left(\begin{array}{c}
\tilde{f}_{n} \\
\tilde{q}_{n}
\end{array}\right)
$$

where the $c_{n}$ 's are real constants, and $\left(\tilde{f}_{n}, \tilde{q}_{n}\right)$ is a normalized eigenfunction of $\hat{L}_{1}$ with eigenvalue $E_{n}$ :

$$
\hat{L}_{1}\left(\begin{array}{c}
\tilde{f}_{n} \\
\tilde{q}_{n}
\end{array}\right)=E_{n}\left(\begin{array}{c}
\tilde{f}_{n} \\
\tilde{q}_{n}
\end{array}\right) .
$$

Then

$$
\delta^{2} \mathcal{F}=\sum_{n} E_{n} c_{n}^{2}
$$

The second variation $\delta^{2} \mathcal{F}$ ceases to be positive-definite when the lowest eigenvalue first becomes negative, indicating that the corresponding solutions $(f, q)$ of the GL equations are unstable. Therefore the entire issue of the stability of the solutions has been reduced to finding the eigenvalue spectrum of the linear stability operator $\hat{L}_{1}$, which in the $\kappa \rightarrow 0$ limit can be studied using matched asymptotic expansions.

Outer solution. The outer equations for $(\tilde{f}, \tilde{q})$ are rescaled with $x^{\prime}=\kappa x$ as before to yield (we will drop the subscript $n$ for notational convenience)

$$
\begin{gathered}
-\tilde{f}^{\prime \prime}+\left(3 f^{2}+q^{2}-1\right) \tilde{f}+2 f q \tilde{q}=E \tilde{f} \\
-\kappa^{2} \tilde{q}^{\prime \prime}+f^{2} \tilde{q}+2 f q \tilde{f}=E \tilde{q}
\end{gathered}
$$

Expanding $\tilde{f}, \tilde{q}$, and $E$ in powers of $\kappa$, and recalling that $q=0$ to all orders in $\kappa$ in the outer region, we have at leading order

$$
-\tilde{f}_{0}^{\prime \prime}+\left(3 f_{0}^{2}-1\right) \tilde{f}_{0}=E_{0} \tilde{f}_{0}
$$

where $f_{0}=\tanh \left(\frac{x^{\prime}+x_{0}}{\sqrt{2}}\right)$. By changing variables to $y=\tanh \left(\frac{x^{\prime}+x_{0}}{\sqrt{2}}\right)$ we see that the solution of Eq. 4.11) is the associated Legendre function of the first kind:

$$
\tilde{f}_{0}\left(x^{\prime}\right)=c_{0} P_{2}^{\mu}\left[\tanh \left(\frac{x^{\prime}+x_{0}}{\sqrt{2}}\right)\right],
$$

where $\mu=-\sqrt{2\left(2-E_{0}\right)}$ and $c_{0}$ is a constant. The leading order solution for $\tilde{q}$ is $\tilde{q}_{0}=0$.

Inner solution. To obtain the inner equations, we rescale as in Eq. (3.14), with the perturbations rescaled as

$$
\tilde{f}\left(x^{\prime}\right)=\tilde{F}(X), \quad \tilde{q}\left(x^{\prime}\right)=\kappa^{-1 / 2} \tilde{Q}(X),
$$

such that

$$
\begin{aligned}
-\frac{1}{\kappa^{2}} \tilde{F}^{\prime \prime}+\left(3 F^{2}+\frac{1}{\kappa} Q^{2}-1\right) \tilde{F}+\frac{1}{\kappa} 2 F Q \tilde{Q} & =E \tilde{F}, \\
-\tilde{Q}^{\prime \prime}+F^{2} \tilde{Q}+2 F Q \tilde{F} & =E \tilde{Q} .
\end{aligned}
$$

To leading order, $\tilde{F}_{0}{ }^{\prime \prime}=0$, so that $\tilde{F}_{0}=a_{0}$, with $a_{0}$ a constant. The leading order equation for the variation in $Q$ is

$$
-\tilde{Q}_{0}^{\prime \prime}+2 F_{0} Q_{0} \tilde{F}_{0}+\left(F_{0}^{2}-E_{0}\right) \tilde{Q}_{0}=0
$$

The solution which satisfies the boundary condition $\tilde{Q}^{\prime}(0)=0$ is

$$
\tilde{Q}_{0}(X)=\frac{2 a_{0} A_{0} B_{0}}{E_{0}}\left(e^{-A_{0} X}-\frac{A_{0}}{\sqrt{A_{0}^{2}-E_{0}}} e^{-\sqrt{A_{0}^{2}-E_{0}} X}\right) .
$$

At $O(\kappa)$ we find 


$$
\tilde{F}_{1}^{\prime \prime}=Q_{0}^{2} \tilde{F}_{0}+2 F_{0} Q_{0} \tilde{Q}_{0}
$$

with the solution

$$
\begin{aligned}
\tilde{F}_{1}(X)=a_{1}+ & a_{0} B_{0}^{2}\left[\frac{E_{0}+4 A_{0}^{2}}{4 A_{0}^{2} E_{0}} e^{-2 A_{0} X}-\frac{4 A_{0}^{2}}{E_{0}} \frac{e^{-\left(A_{0}+\sqrt{A_{0}^{2}-E_{0}}\right) X}}{\left(A_{0}+\sqrt{A_{0}^{2}-E_{0}}\right)^{2} \sqrt{A_{0}^{2}-E_{0}}}\right] \\
+ & a_{0} B_{0}^{2}\left[\frac{E_{0}+4 A_{0}^{2}}{2 A_{0} E_{0}}-\frac{4 A_{0}^{3} / E_{0}}{\left(A_{0}+\sqrt{A_{0}^{2}-E_{0}}\right) \sqrt{A_{0}^{2}-E_{0}}}\right] X .
\end{aligned}
$$

We now have enough terms in the inner and outer region for a nontrivial match.

Matching. We complete the matching of the inner and outer perturbations to obtain the eigenvalue, $E_{0}$. Performing a two term inner expansion of the one term outer solution, we have

$$
\tilde{f}_{0}(\kappa X) \sim c_{0}\left[P_{2}^{\mu}\left(A_{0}\right)+\frac{1}{\sqrt{2}} \operatorname{sech}^{2}\left(x_{0} / \sqrt{2}\right) \frac{d P_{2}^{\mu}\left(A_{0}\right)}{d A_{0}} \kappa X\right],
$$

where we have used $\tanh \left(x_{0} / \sqrt{2}\right)=A_{0}$. Next, the one term outer expansion of the two term inner solution is

$$
\tilde{F}_{0}\left(x^{\prime} / \kappa\right)+\kappa \tilde{F}_{1}\left(x^{\prime} / \kappa\right) \sim a_{0}+\frac{a_{0} 2^{1 / 2}\left(1-A_{0}^{2}\right)}{E_{0}}\left[\frac{E_{0}+4 A_{0}^{2}}{2 A_{0}}-\frac{4 A_{0}^{3}}{\left(A_{0}+\sqrt{A_{0}^{2}-E_{0}}\right) \sqrt{A_{0}^{2}-E_{0}}}\right] x^{\prime},
$$

where we have used $B_{0}=-2^{1 / 4}\left(1-A_{0}^{2}\right)^{1 / 2}$. Matching the two expansions using the van Dyke matching principle yields

$$
\begin{gathered}
c_{0}=\frac{a_{0}}{P_{2}^{\mu}\left(A_{0}\right)}, \\
\frac{1}{P_{2}^{\mu}\left(A_{0}\right)} \frac{d P_{2}^{\mu}\left(A_{0}\right)}{d A_{0}}=\frac{2}{E_{0}}\left[\frac{E_{0}+4 A_{0}^{2}}{2 A_{0}}-\frac{4 A_{0}^{3}}{\left(A_{0}+\sqrt{A_{0}^{2}-E_{0}}\right) \sqrt{A_{0}^{2}-E_{0}}}\right] .
\end{gathered}
$$

The last equation is a rather complicated implicit equation for the eigenvalue $E_{0}\left(A_{0}\right)$, which generally must be solved numerically. However, when $A_{0}=1 / \sqrt{2}$ we find $E_{0}=0$, corresponding to the critical case, with $E>0$ for $A_{0}>1 / \sqrt{2}$. The numerical evaluation of Eq. (4.23) is shown in Fig. 5. Therefore, we see that our maximum superheating field (at lowest order) corresponds to the limit of metastability for these one-dimensional perturbations. In Fig. 6 we show $A_{0}$ as a function of the lowest order magnetic field at the surface, $H_{0}$, from Eq. (3.38). The stability analysis of this section shows that only the upper branch of this double valued function corresponds to solutions which are locally stable, with the field at the "nose" being the superheating field.

\section{B. Stability with respect to two-dimensional perturbations}

We next turn to the stability of the solutions with respect to two dimensional perturbations. If we perturb the extremal solution $(f, \mathbf{q})$ of the GL equations by allowing $f \rightarrow f+\delta f$ and $\mathbf{q} \rightarrow \mathbf{q}+\delta \mathbf{q}$, then the second variation of the free energy functional is

$$
\delta^{2} \mathcal{F}=\int d x d y\left[\frac{1}{\kappa^{2}}(\nabla \delta f)^{2}+4 f(\delta f) \mathbf{q} \cdot \delta \mathbf{q}+f^{2}(\delta \mathbf{q})^{2}+\left(3 f^{2}+\mathbf{q}^{2}-1\right)(\delta f)^{2}+(\nabla \times \delta \mathbf{q})^{2}\right]
$$

(we neglect perturbations along the $z$-direction). Expanding in Fourier modes with respect to $y$,

$$
\delta f(x, y)=\tilde{f}(x) \cos k y, \quad \delta q_{x}(x, y)=\tilde{q}_{x}(x) \sin k y, \quad \delta q_{y}(x, y)=\tilde{q}_{y}(x) \cos k y,
$$

substituting into Eq. (4.24), recalling that $\mathbf{q}=(0, q(x), 0)$, and integrating over $y$, we obtain (up to a multiplicative constant)

$$
\delta^{2} \mathcal{F}=\int_{0}^{\infty} d x\left[\frac{1}{\kappa^{2}} \tilde{f}^{\prime 2}+\left(3 f^{2}+q^{2}+\frac{1}{\kappa^{2}} k^{2}-1\right) \tilde{f}^{2}+4 f q \tilde{f} \tilde{q}_{y}+f^{2}\left(\tilde{q}_{x}^{2}+\tilde{q}_{y}^{2}\right)+\left(\tilde{q}_{y}^{\prime}-k \tilde{q}_{x}\right)^{2}\right] .
$$


By integrating by parts and using the boundary conditions, Eq. (4.2), we can cast this functional into the form

$$
\delta^{2} \mathcal{F}=\int_{0}^{\infty} d x\left(\tilde{f}, \tilde{q}_{y}, \tilde{q}_{x}\right) \hat{L}_{2}\left(\begin{array}{c}
\tilde{f} \\
\tilde{q}_{y} \\
\tilde{q}_{x}
\end{array}\right)
$$

where the self-adjoint linear operator $\hat{L}_{2}$ is given by

$$
\hat{L}_{2}\left(\begin{array}{c}
\tilde{f} \\
\tilde{q}_{y} \\
\tilde{q}_{x}
\end{array}\right)=\left(\begin{array}{ccc}
-\frac{1}{\kappa^{2}} \frac{d^{2}}{d x^{2}}+q^{2}+3 f^{2}+k^{2} / \kappa^{2}-1 & 2 f q & 0 \\
2 f q & -\frac{d^{2}}{d x^{2}}+f^{2} & -k \frac{d}{d x} \\
0 & k \frac{d}{d x} & f^{2}+k^{2}
\end{array}\right)\left(\begin{array}{c}
\tilde{f} \\
\tilde{q}_{y} \\
\tilde{q}_{x}
\end{array}\right) .
$$

As in the previous section, we want to determine the eigenvalue spectrum of this operator. We are primarily interested in the effects of long-wavelength perturbations (i.e., $k \rightarrow 0$ ), so we rescale $k$ as $k=\kappa k^{\prime}$. Then the eigenvalue equations in terms of the outer coordinate $x^{\prime}=\kappa x$ are (dropping the prime on $k$ from now on)

$$
\begin{gathered}
-\tilde{f}^{\prime \prime}+\left(3 f^{2}+q^{2}-1+k^{2}\right) \tilde{f}+2 f q \tilde{q}=E \tilde{f} \\
-\kappa^{2} \tilde{q}_{y}^{\prime \prime}+f^{2} \tilde{q}_{y}+2 f q \tilde{f}-\kappa^{2} k \tilde{q}_{x}^{\prime}=E \tilde{q}_{y} \\
\kappa^{2} k \tilde{q}_{y}^{\prime}+\left(f^{2}+\kappa^{2} k^{2}\right) \tilde{q}_{x}=E \tilde{q}_{x}
\end{gathered}
$$

By using the last equation we may eliminate $\tilde{q}_{x}$ from Eq. (4.30), which becomes

$$
-\kappa^{2} \frac{d}{d x}\left[\frac{f^{2}-E}{f^{2}+\kappa^{2} k^{2}-E} \tilde{q}_{y}^{\prime}\right]+f^{2} \tilde{q}_{y}+2 f q \tilde{f}=E \tilde{q}_{y} .
$$

For $k=0$ Eqs. (4.29) and (4.32) reduce to the one-dimensional perturbation equations of the last section, Eqs. (4.9) and (4.10); for $E=0$ they reduce to the Euler-Lagrange equations derived by Kramer [8].

The perturbation equations (4.29) and 4.32) may be solved by the method of matched asymptotic expansions, just as in the one-dimensional case. The derivation of the eigenvalue condition is essentially identical, with the final result that

$$
\frac{1}{P_{2}^{\mu}\left(A_{0}\right)} \frac{d P_{2}^{\mu}\left(A_{0}\right)}{d A_{0}}=\frac{2}{E_{0}}\left[\frac{E_{0}+4 A_{0}^{2}}{2 A_{0}}-\frac{4 A_{0}^{3}}{\left(A_{0}+\sqrt{A_{0}^{2}-E_{0}}\right) \sqrt{A_{0}^{2}-E_{0}}}\right]
$$

where now $\mu=-\sqrt{2\left(2+E_{0}-k^{2}\right)}$. The eigenvalue $E_{0}(k)$ is plotted in Fig. 7 for several different values of $A_{0}$. For $A_{0}>1 / \sqrt{2}, E_{0}(k)>0$ for all $k$, while for $A_{0}<1 / \sqrt{2}$ there exists a band of long-wavelength perturbations for which $E_{0}(k)<0$. In all cases the most unstable modes are at $k=0$, i.e., the one-dimensional perturbations are the least stable. This is in contrast to the large- $\kappa$ limit, where the most unstable mode occurs for $k \neq 0$ [17,8, 15.

\section{NONLOCAL EFFECTS AS $\kappa \rightarrow 0$}

In the previous sections we have studied superheating in type-I superconductors starting from the conventional GL equations, which assume a local relationship between the current and the vector potential. However, in very clean type-I superconductors nonlocal effects are often important (in the Pippard limit; see Ref. [18]). We can model these effects by replacing the second GL equation, Eq. (3.2), by a nonlocal equation of the form

$$
\kappa^{2} q^{\prime \prime}-\int_{0}^{\infty} K\left(x-x^{\prime}\right) f^{2}\left(x^{\prime}\right) q\left(x^{\prime}\right) d x^{\prime}=0,
$$

where $K\left(x-x^{\prime}\right)$ is a kernel whose Fourier transform $K(k)$ behaves as

$$
K(k)= \begin{cases}\lambda^{2} / \lambda_{L}^{2} & \text { (local limit); } \\ a /|k| & \text { (extreme anomalous limit) }\end{cases}
$$


with $\lambda_{L}$ the London penetration depth and $a$ a constant 18 . For $\lambda \approx \lambda_{L}$ we recover the local limit considered in the previous sections of this paper. It is still possible to calculate the superheating field in this nonlocal limit using the method of matched asymptotic expansions. Indeed, the prescription is the same as for the local case discussed above; we only need to solve a slightly more complicated inner problem. In this section we will calculate the leading order superheating field in the nonlocal limit, in order to further illustrate the power and flexibility of our method.

Outer solution. The outer solution is the same as before; the vector potential is zero to all orders, and the first two terms in the expansion for the order parameter are given by Eqs. (3.9) and (3.13).

Inner solution. In the inner region we rescale the variables as in Eq. (3.14). In terms of these variables Eq. (5.1) becomes

$$
Q^{\prime \prime}-\int_{0}^{\infty} K\left(X-X^{\prime}\right) F^{2}\left(X^{\prime}\right) Q\left(X^{\prime}\right) d X^{\prime}=0 .
$$

We need to solve this equation, along with the first GL equation, Eq. (3.15), perturbatively in $\kappa$. Expanding $F$ and $Q$ as in Eqs. (3.19)-(3.21), we obtain $F_{0}(X)=A_{0}$, as before, and

$$
Q_{0}^{\prime \prime}-A_{0}^{2} \int_{0}^{\infty} K\left(X-X^{\prime}\right) Q_{0}\left(X^{\prime}\right) d X^{\prime}=0 .
$$

This is an integral equation of the Wiener-Hopf type [19. To solve, we Fourier transform, introducing

$$
Q_{+}(k)=\int_{0}^{\infty} Q_{0}(X) e^{i k X} d X
$$

After Fourier transforming the integral equation, we perform a Wiener-Hopf factorization [19], with the result that

$$
Q_{+}(k)=B_{0} \frac{i e^{i \varphi(k)}}{\left[k^{2}+A_{0}^{2} K(k)\right]^{1 / 2}},
$$

where $B_{0}=Q_{0}(0)$ is a constant, and

$$
\varphi(k)=\frac{k}{\pi} \int_{0}^{\infty} \ln \left[\frac{k^{2}+A_{0}^{2} K(x)}{x^{2}+A_{0}^{2} K(k)}\right] \frac{1}{x^{2}-k^{2}} d x .
$$

The Fourier transform can be inverted once a particular form for $K(k)$ is specified (although this is unnecessary for the calculation of the superheating field; see below). The magnetic field at this order is $H_{0}(0)=-A_{0} B_{0}$ as before.

Proceeding to the next order, we have

$$
F_{1}^{\prime \prime}=A_{0} Q_{0}^{2}
$$

By applying the boundary condition $F_{1}^{\prime}(0)=0$, we find the general solution

$$
F_{1}(X)=A_{1}+A_{0}\left[X \int_{0}^{X} Q_{0}^{2}(y) d y-\int_{0}^{X} y Q_{0}^{2}(y) d y\right]
$$

with $A_{1}=F_{1}(0)$ another constant. The equation for $Q_{1}(X)$ is a rather messy inhomogeneous Wiener-Hopf integral equation. Fortunately, its solution is not needed for the leading order calculation of the superheating field.

Matching. We now turn to the matching of the inner and outer solutions. The two term inner expansion of the one term outer solution is

$$
f_{0}(\kappa X) \sim \tanh \left(\frac{x_{0}}{\sqrt{2}}\right)+\frac{\kappa}{\sqrt{2}} \operatorname{sech}^{2}\left(\frac{x_{0}}{\sqrt{2}}\right) X .
$$

The one term outer expansion of the two term inner solution is

$$
F_{0}\left(x^{\prime} / \kappa\right)+\kappa F_{1}\left(x^{\prime} / \kappa\right) \sim A_{0}+A_{0}\left(\int_{0}^{\infty} Q_{0}^{2}(y) d y\right) x^{\prime} .
$$

By using the van Dyke matching principle we find $A_{0}=\tanh \left(x_{0} / \sqrt{2}\right)$ as before, and 


$$
A_{0} \int_{0}^{\infty} Q_{0}^{2}(y) d y=\frac{1}{\sqrt{2}}\left(1-A_{0}^{2}\right) .
$$

We can use Parseval's identity to express the left hand side of Eq. (5.12) in terms of $\left|Q_{+}(k)\right|^{2}$, and then use Eq. (5.6) to finally arrive at

$$
\frac{A_{0} B_{0}^{2}}{2 \pi} \int_{-\infty}^{\infty} \frac{1}{k^{2}+A_{0}^{2} K(k)} d k=\frac{1}{\sqrt{2}}\left(1-A_{0}^{2}\right) .
$$

To calculate the superheating field we use Eq. (5.13) to express $B_{0}$ as a function of $A_{0}$; we then substitute this result into $H_{0}(0)=-A_{0} B_{0}$ and maximize with respect to $A_{0}$ in order to determine the lowest order superheating field. In the local limit, $K(k)=\lambda^{2} / \lambda_{L}^{2}$, and we obtain $A_{0}^{*}=1 / \sqrt{2}$ and $H_{0}(0)=2^{-3 / 4}\left(\lambda_{L} / \lambda\right)$, which is the same as our previous result when $\lambda \approx \lambda_{L}$. In the extreme anomalous limit $K(k)=a /|k|$, with $a=(3 \pi / 4)\left(\lambda^{2} \xi / \lambda_{L}^{2} \xi_{0}\right)$ in the Pippard theory [18], where $\xi_{0}$ is the zero temperature coherence length. Performing the integral, we find

$$
B_{0}=-3^{3 / 4} 2^{-3 / 4} a^{1 / 6} A_{0}^{-1 / 6}\left(1-A_{0}^{2}\right)^{1 / 2},
$$

so that

$$
H_{0}(0)=3^{3 / 4} 2^{-3 / 4} a^{1 / 6} A_{0}^{5 / 6}\left(1-A_{0}^{2}\right)^{1 / 2} .
$$

The maximum occurs at $A_{0}^{*}=\sqrt{5 / 11}$, so that $H_{0}(0)=0.721 a^{1 / 6}$. Therefore, the superheating field is

$$
H_{\mathrm{sh}}=0.721 a^{1 / 6} \kappa^{-1 / 2}+O\left(\kappa^{1 / 2}\right) \quad \text { (extreme anomalous limit). }
$$

The same result has been obtained by Smith et al. 20] using an approximation for the order parameter along with a variational calculation (in the spirit of method used by the Orsay group [5]). The advantage of our method is that it can be systematically improved upon. Although we have not checked the stability in the extreme anomalous limit, the procedure should be entirely analogous to that of the previous section.

\section{LARGE- $\kappa$ RESULTS}

So far we have used the method of matched asymptotics to solve the GL equations in the small- $\kappa$ limit. Chapman 15] has recently used the same method to treat the one dimensional GL equations in the high- $\kappa$ limit. His final result for the superheating field is

$$
H_{\mathrm{sh}}=\frac{1}{\sqrt{2}}+C \kappa^{-4 / 3}+O\left(\kappa^{-6 / 3}\right)
$$

where the constant $C$ is determined from the solution of the second Painlevè transcendent; a numerical evaluation yields $C=0.326$ [21]. The first term was originally derived by Ginzburg [4], and the second term with the unusual dependence upon $\kappa$ is the new term. As seen in Fig 8 the asymptotic and numerical results agree very well. It turns out, however, that the calculated $H_{\text {sh }}$ is not actually the superheating field, since the one dimensional solution in the large- $\kappa$ limit is unstable with respect to two-dimensional perturbations [17,8, 15]; these instabilities occur at at the smaller field $H_{\mathrm{sh}}^{2 D}=\sqrt{5} / 3 \sqrt{2}=0.527$. This situation is quite different from that of the small- $\kappa$ limit in which our stability calculation (Section IV) found the limit of stability to be right at $H_{\mathrm{sh}}$.

\section{DISCUSSION}

In this paper, the one-dimensional GL equations are solved analytically and numerically for a semi-infinite superconducting sample in the small- $\kappa$ limit in order to determine the maximum superheating field $H_{\mathrm{sh}}$. We have used the method of matched asymptotic expansions to construct for the first time a systematic perturbative solution of the Ginzburg-Landau equations, the results of which agree quite closely with our numerical solutions. The same method has been used to determine the stability of these solutions with respect to both one- and two-dimensional infinitesimal fluctuations; our analysis shows that two dimensional fluctuations do not lead to any additional destabilizing effects, in contrast to the situation in the large- $\kappa$ limit. With little modification this method can also be adapted to treat nonlocal electrodynamic effects. Finally, our numerical results for large- $\kappa$ compare well with Chapman's asymptotic analysis of this regime. Taken collectively, our results demonstrate the effectiveness of the method of matched asymptotic expansions for dealing with boundary layer problems in the theory of superconductivity. We hope that others will find useful applications of the methods developed in this paper in treating inhomogeneous superconductors. 


\section{ACKNOWLEDGMENTS}

We would like to thank Dr. Chung-Yu Mou for helpful discussions, Dr. S. J. Chapman for useful correspondence, and Dr. C. Bolley for sending us her preprints and reprints on superheating in superconductors. This work was supported by NSF Grant DMR 92-23586, as well as by the Alfred P. Sloan Foundation (ATD).

$1 \quad$ Electronic address: ajd2m@virginia.edu

$2 \quad$ Electronic address: sjd2u@virginia.edu

$3 \quad$ Electronic address: atd2h@virginia.edu

[1] See, for instance, A. Barone (ed.), Superconductive Particle Detectors (World Scientific, Singapore, 1987); K. Pretzl, J. Low Temp. Phys. 93, 439 (1993), and the subsequent articles in the same volume.

[2] For instance, see J. P. Burger and D. Saint-James, in Superconductivity, Vol. 2, R. D. Parks, ed. (Marcel Dekker, New York, 1969), Ch. 16.

[3] This criteria is well satisfied in practice. See P. G. de Gennes, Superconductivity in Metals and Alloys (Addison-Wesley, New York, 1966), Ch. 7.

[4] V. L. Ginzburg, Sov. Phys. JETP 7, 78 (1958).

[5] The Orsay group, in Quantum Fluids (ed. D. F. Brewer), p. 26 (North Holland, Amsterdam 1966).

[6] H. Parr, Z. Phys. B 25, 359 (1976).

[7] J. Matricon and D. Saint-James, Phys. Lett. 24A, 241 (1967).

[8] L. Kramer, Phys. Rev. 170, 475 (1968).

[9] H. J. Fink and A. G. Presson, Phys. Rev. 182, 498 (1969).

[10] C. Bolley and B. Helffer, "Superheating in a film in the weak $\kappa$ limit: numerical results and approximate models," Ecole Centrale Nantes (preprint 1994).

[11] For a review of the numerical work on superheating fields in superconductors, see H. J. Fink, D. S. McLachlan, and B. Rothberg Bibby, in Progress in Low Temperature Physics, Vol. VII B, D. F. Brewer, ed. (North-Holland Publishing, Amsterdam, 1978).

[12] V. P. Galaiko, Zh. Eksp. Teor. Fiz. 54, 318 (1968) [Sov. Phys. JETP 27, 170 (1968)].

[13] C. M. Bender and S. A. Orszag, Advanced Mathematical Methods for Scientists and Engineers (McGraw-Hill, New York, 1978).

[14] M. van Dyke, Perturbation Methods in Fluid Mechanics (Parabolic Press, Stanford CA, 1975).

[15] S. J. Chapman, "Superheating field of type-II superconductors," Mathematical Institute, Oxford (preprint 1994).

[16] W. H. Press, S. A. Teukolsky, W. T. Vetterling, and B. P. Flannery, Numerical Recipes in FORTRAN, Second Edition (Cambridge University Press, Cambridge, UK, 1992), Ch. 17.

[17] V. P. Galaiko, Zh. Eksp. Teor. Fiz. 50, 717 (1966) [Sov. Phys. JETP 23, 475 (1966)].

[18] M. Tinkham, Introduction to Superconductivity (McGraw-Hill, New York, 1975), Ch. 3.

[19] For a general introduction to Wiener-Hopf integral equations, see G. F. Carrier, M. Krook, and C. E. Pearson, Functions of a Complex Variable (Hod Books, Ithaca, New York, 1983), pp. 376-432. For a recent application of the Wiener-Hopf method to the solution of an integral equation which arises in the theory of superconducting thin-films, see A. T. Dorsey, Phys. Rev. B 51, 15329 (1995).

[20] F. W. Smith, A. Baratoff, and M. Cardona, Phys. Kodens. Materie 12, 145 (1970).

[21] A. Dolgert and S. J. Di Bartolo (unpublished).

FIG. 1. A comparison of the numerically calculated superheating field $H_{\text {sh }}$ (heavy line) with the three term asymptotic expansion for small- $\kappa$, and the [2,2] Padé approximant. The one-term expansion due to the Orsay group deviates systematically from the calculated superheating field. The two- and three-term expansions provide a marked improvement over the one-term expansion.

FIG. 2. A comparison of the three term inner and outer solutions for the order parameter and the magnetic field with the numerical solution for $\kappa=0.1$. The asymptotic solutions approximate the computed values only in the appropriate regions. The matching region where the inner and outer meet is $O(\kappa)$ as can be estimated from the inner solution for $f$.

FIG. 3. The same as Fig. 目 for $\kappa=0.5$. 
FIG. 4. A comparison of the two-term uniform solution for the order parameter, $f_{\text {unif }, 2}(x)$ (dashed line), with the numerical solution (solid line) at $\kappa=0.5$. The disagreement of the uniform solution with the boundary condition at $x=0$ is of order $\kappa^{2}$.

FIG. 5. The stability eigenvalue $E\left(A_{0}\right)$, with $A_{0}$ the value of the order parameter at the surface at leading order. We see that $E>0$ for $A_{0}>1 / \sqrt{2}$, indicating locally stable solutions.

FIG. 6. The order parameter at the surface, $A_{0}$, as a function of the field at the surface, $H_{0}$, at leading order. The stability analysis shows that only the upper branch corresponds to locally stable solutions. The field at the "nose" is the limit of stability, and corresponds to the superheating field $H_{0}=2^{-3 / 4}=0.595$.

FIG. 7. The stability eigenvalue $E(k)$ for two-dimensional perturbations of wavenumber $k$, for several different values of $A_{0}$. For $A_{0}>1 / \sqrt{2}$ the eigenvalue is stable for all wavenumbers, while for $A_{0}<1 / \sqrt{2}$ there exists a band of wavenumbers for which the solution is unstable.

FIG. 8. The numerically calculated superheating field for large- $\kappa$ (solid line), compared with the two-term asymptotic expansion derived by Chapman (dashed line). The slope of the dashed line is $-4 / 3$.

TABLE I. Summary of the results of the small- $\kappa$ expansion for the superheating field. Here $A_{n}$ is the value of the order parameter $F(X)$ at the surface at $n$-th order, $B_{n}$ is the value of the vector potential $Q(X)$ at $n$-th order, $C_{n}$ is the coefficient of the $n$-th term in the outer expansion of the order parameter, and $H_{n}(0)$ is the $n$-th order term in the expansion of the superheating field.

\begin{tabular}{lcccc}
\hline \hline$n$ & $A_{n}$ & $B_{n}$ & $C_{n}$ & $H_{n}(0)$ \\
\hline 0 & $2^{-1 / 2}$ & $-2^{-1 / 4}$ & 1 & $2^{-3 / 4}$ \\
1 & $-7 / 32$ & $-(9 / 16) 2^{1 / 4}$ & $-(15 / 16) 2^{1 / 2}$ & $(15 / 64) 2^{3 / 4}$ \\
2 & $-(17 / 1024) 2^{1 / 2}$ & $(159 / 2048) 2^{3 / 4}$ & $225 / 256$ & $-(325 / 2048) 2^{1 / 4}$ \\
3 & $3211 / 16384$ & $-(745 / 4096) 2^{1 / 4}$ & $-(1125 / 4096) 2^{1 / 2}$ & $(14191 / 65536) 2^{3 / 4}$ \\
4 & $-(623575 / 1572864) 2^{1 / 2}$ & $(16223049 / 20971520) 2^{3 / 4}$ & $16875 / 131072$ & $-(78495727 / 62914560) 2^{1 / 4}$ \\
& & & \\
\hline \hline
\end{tabular}




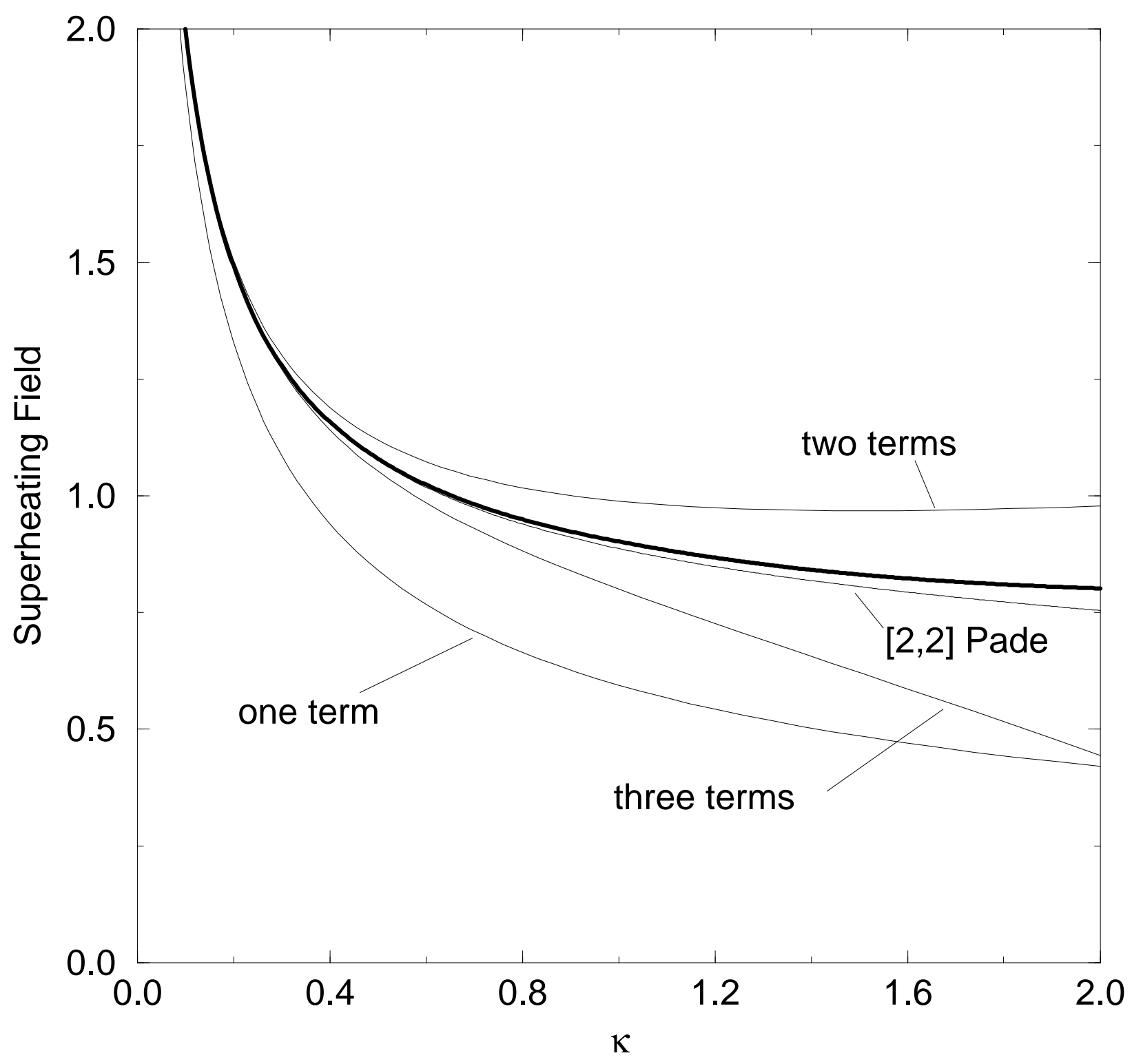



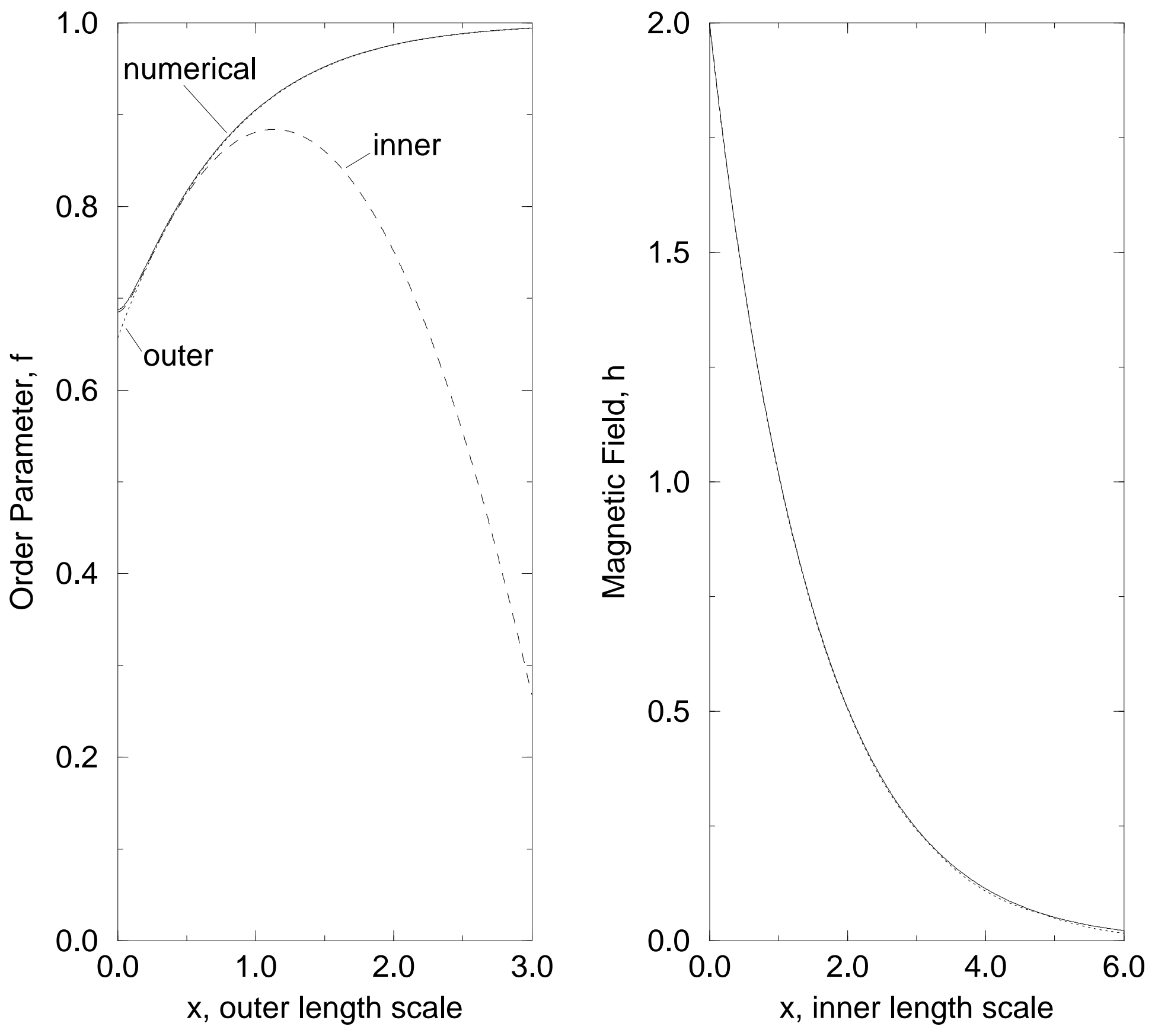




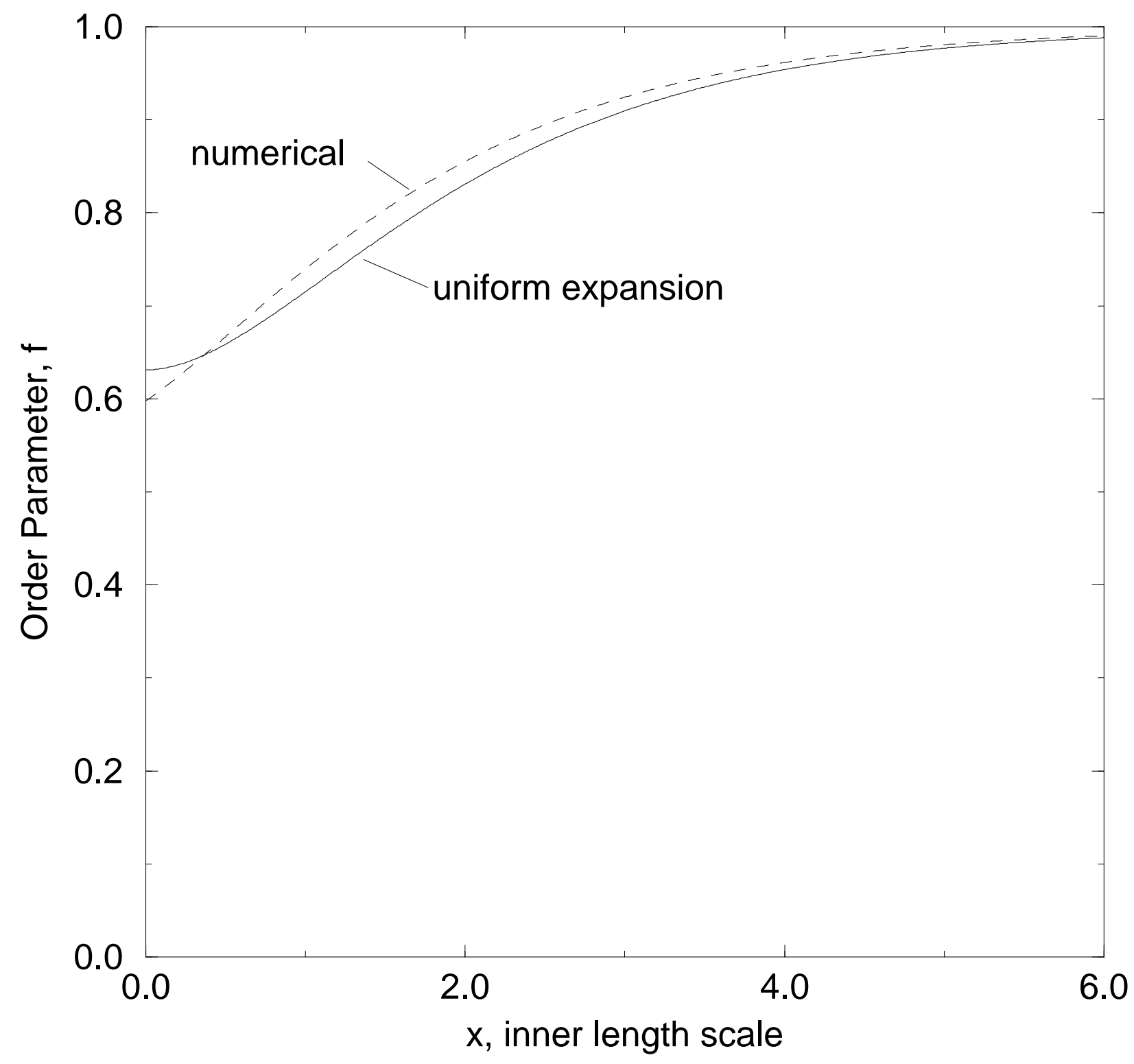




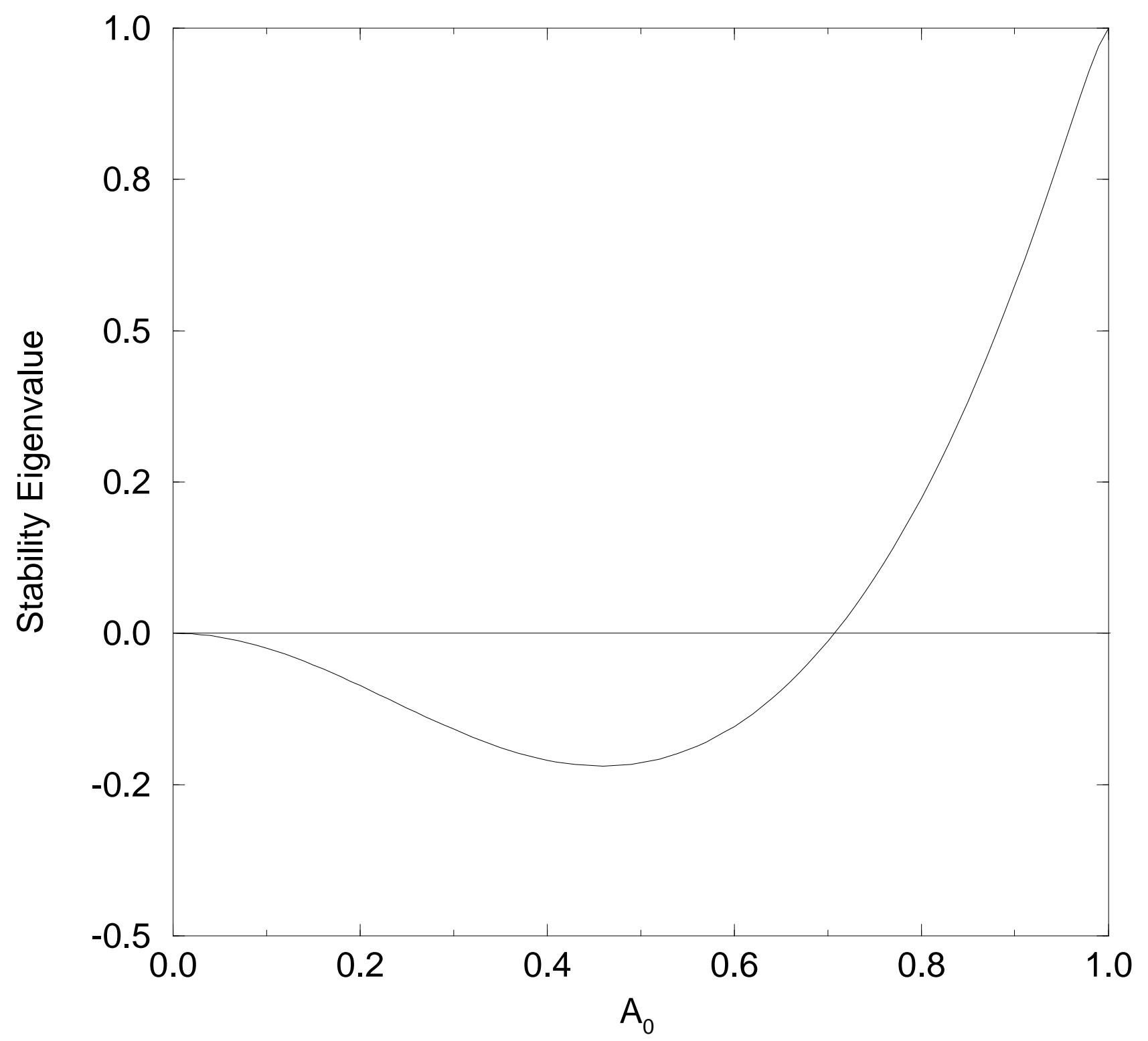




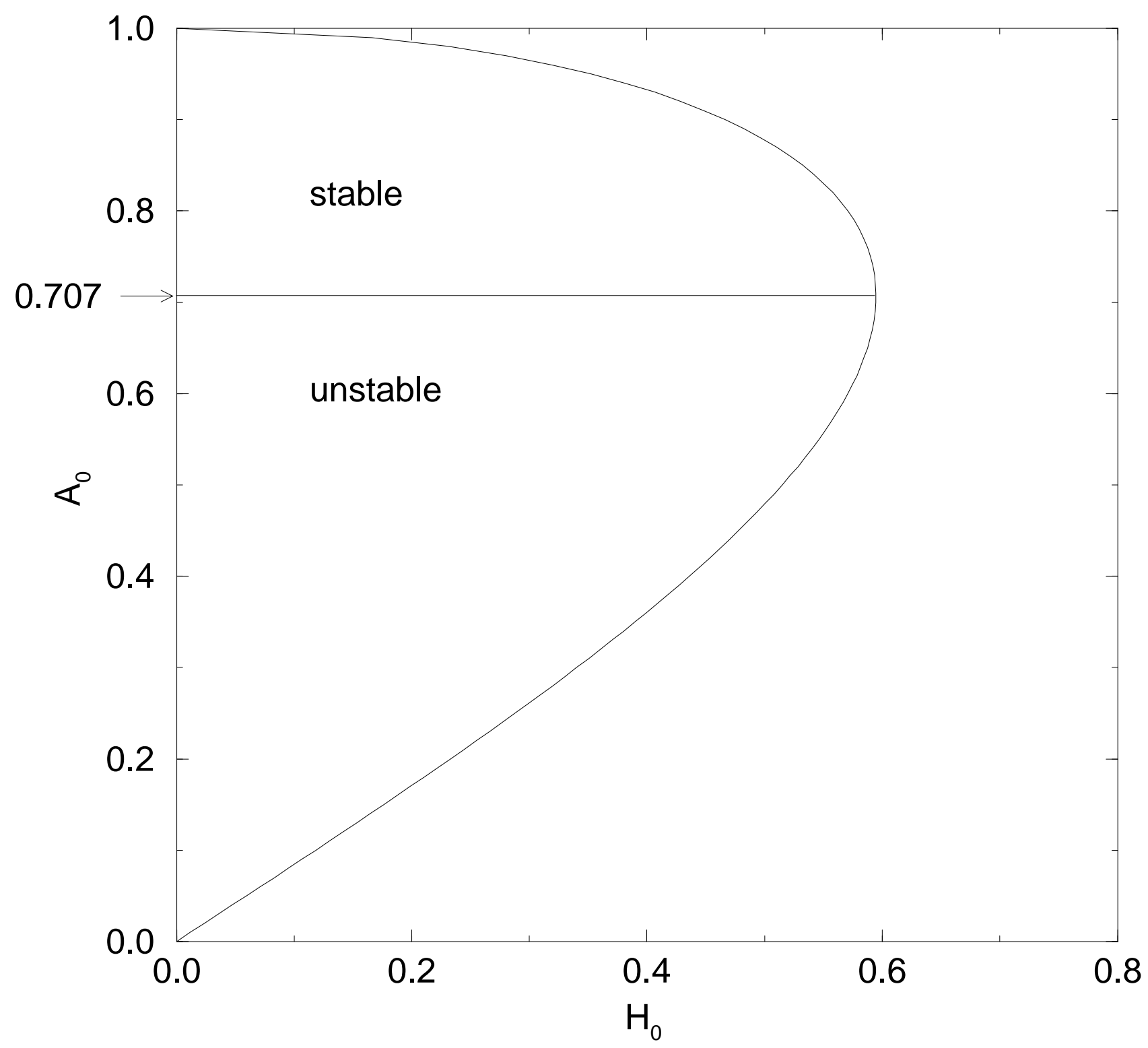




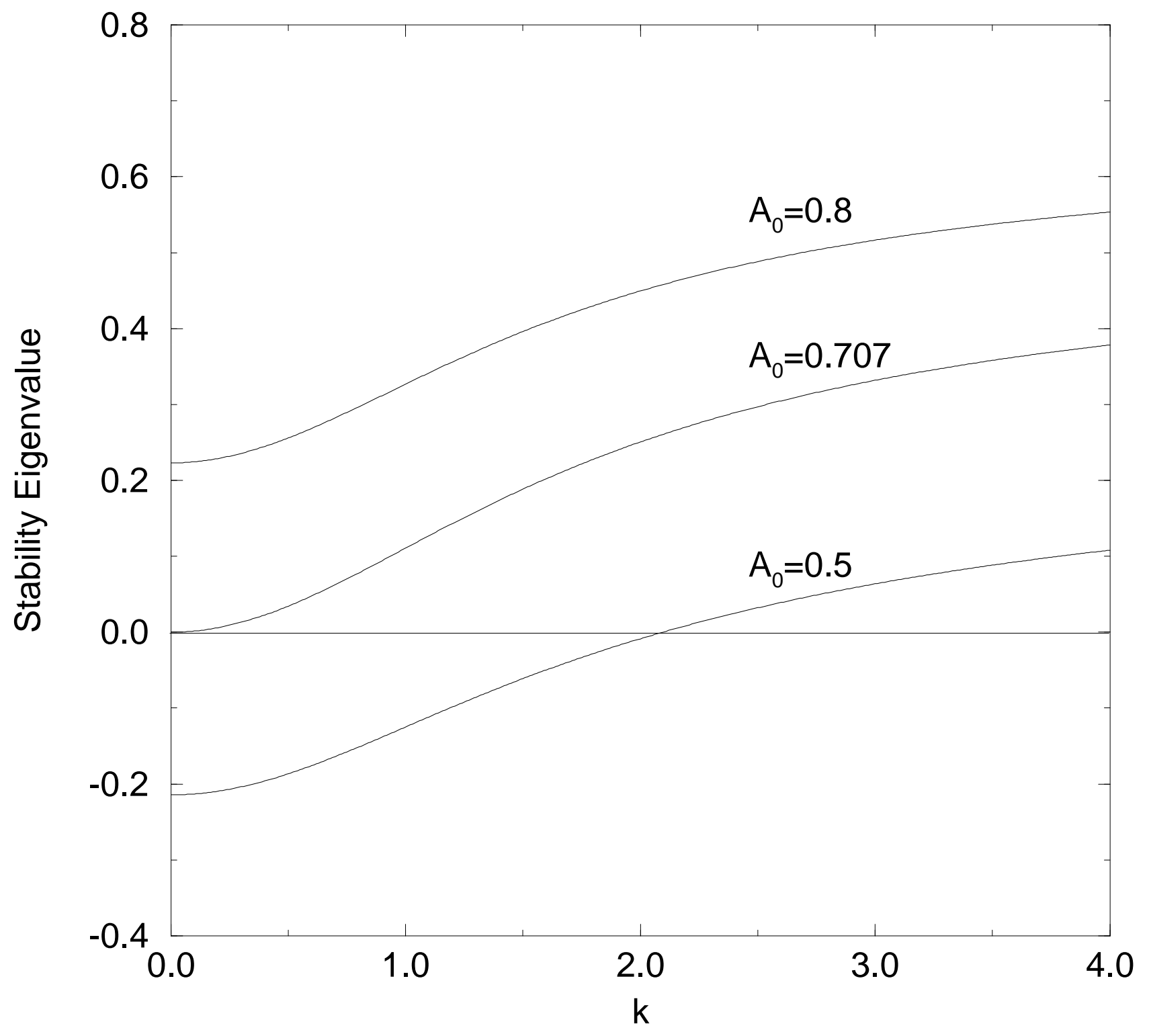




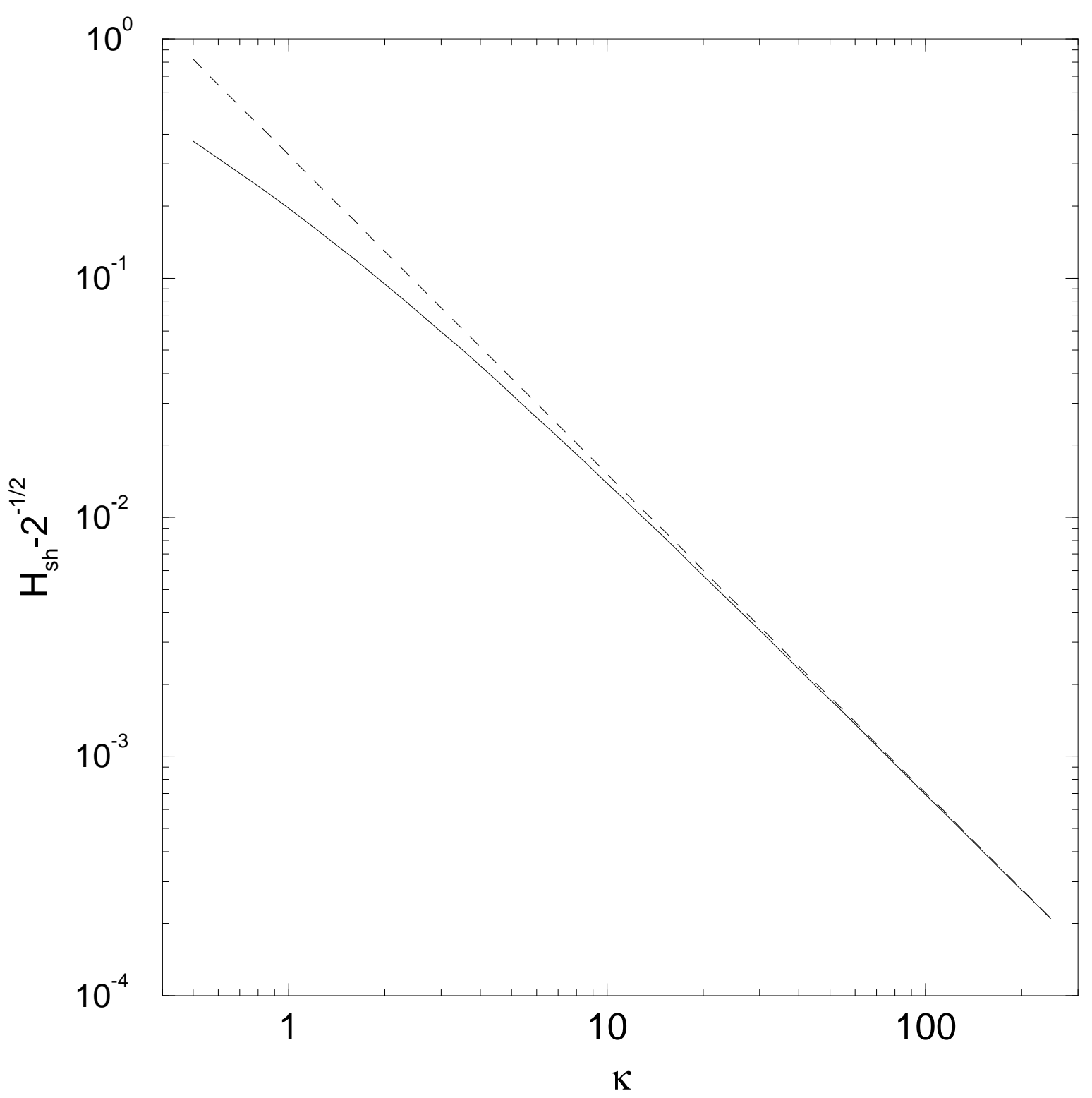

